\title{
OPTIMAL DESIGN OF A VIBRATION ABSORBER-HARVESTER DYNAMIC SYSTEM
}

\author{
Galal A. Hassaan ${ }^{1}$ \\ ${ }^{I}$ Emeritus Professor, Department of Mechanical Design and Production, Faculty of Engineering, Cairo University, \\ Giza, EGYPT
}

\begin{abstract}
Vibration dynamic absorber is in use to reduce machinery vibrations. This paper presents a novel idea for using the mass-spring absorber as an energy harvester. The aim is to tune the absorber to cancel the machine vibration and produce maximum vibration of the absorber mass to act as an energy harvester. The main vibrating system is damped and assumed as a SDOF. A new frequency is defined called harvesting frequency at which the amplitude of the absorber mass vibration is maximum. It is shown that his frequency is dependent only on the mass ratio of the absorber and independent of the main system damping ratio. The optimal operation of the proposed system is investigated for damping ratio between 0.1 and 0.4 and mass ratio between 0.05 and 4.5. The absorber succeeds to eliminate completely the main system vibrations at a tuning condition and provides high vibration amplitude at the absorber mass at the new defined harvesting frequency..
\end{abstract}

Keywords: Vibration absorber, Energy harvester, Harvesting frequency, Absorber tuning

\section{INTRODUCTION}

Vibration control is required to reduce machinery and structural vibrations to levels accepted by international standards. One of the techniques used in vibration control is using dynamic vibration absorbers. A lot of research works have been carried out during the last decades. We focus only in this introduction on some research work in the last 15 years. Pade and Steffen (2000) studied the reduction of vibration levels of machinery systems using dynamic vibration absorbers. They proposed a general methodology for the optimal selection of the absorber parameters to guarantee their effectiveness over a frequency band [1]. Miller (2003) investigated the nonlinear mechanical properties of elastomers in reference to their potential application in adaptive-passive tuned vibration absorbers. He focused on changing the absorber natural frequency in the range 45 to $211 \mathrm{~Hz}$ [2]. Bonsel, Fey and Nijmeijer (2004) studied the application of a linear dynamic vibration absorber to a piecewise linear beam system to suppress its first resonance. They considered both the undamped and damped absorbers [3]. Varpasuo (2006) studied the response characteristics of stochastic vibration absorber. He assessed the effectiveness of the vibration absorber for various stiffness and damping configurations [4]. Jang et.al. (2007) studied the design of a cantilever type multi-DOF vibration absorber. They used three cantilever beams and a rigid body suspended from the beam as a spring-mass system to reduce resonant vibrations of the main system [5]. Ozkan (2010) studied the application of passive tuned mass damper (single or multiple) to Euler-Bernoulli beams and examined their effectiveness base on free and forced vibration characteristics of the beams [6]. Liao et.al. (2011) presented an active-adaptive tuned vibration absorber based on magneto rheological elastomer. They studied two feedback types of activation force and incorporated phase-lead compensator to eliminate the time delay effect during signal processing [7]. Mirsanei et.al. (2012) presented a design for an adaptive tuned dynamic vibration absorber based on a smart slider-crank mechanism [8]. Shen and Ahmadian (2013) studied analytically four semi-active dynamic vibration absorbers including the time delay induced by measurement and execution. They discussed the effect of time delay on the control performance [9]. Huang and Lin (2014) designed a vibration absorber called periodic vibration absorber for mechanical systems subjected to periodic excitation. They claimed that their design can absorb significant amount of higher harmonics in addition to the fundamental harmonic [10].

Energy harvesting is relatively a new topic aiming at discovering new sources of clean electric energy to avoid environment pollution from the classical oil-based techniques. Here, I focus of some of the efforts during the last five years. Kim et.al. (2010) designed a piezoelectric energy harvester aiming at increasing the output electric power efficiency by analyzing a finite element method for the structure of the energy harvester [11]. Li, Yuan and Lipson (2011) studied experimentally a piezo-leaf architecture converting wind energy into electrical energy through a wind-induced fluttering motion [12]. Han, Shin and Choi ( 2012) presented a wideband vibration system of an electromagnetic vibration energy harvester of $2 \mathrm{DOF}$ vibrations [13]. Wang, Chen and Sung (2013) proposed a system design of a weighted pendulum-type electromagnetic operator for harvesting energy from a rotating wheel. They discussed the nonlinear dynamic behavior of the system and aimed to have large pendulum angle and velocity to generate large amount of power [14]. Nithiya, Sadhuri and Saravanan (2014) developed a pendulum motion based power generator to be used with reciprocating mechanical structures. They 
aimed to produce a platform of developing a sustainable and low maintenance system to harvest electrical energy [15].

\section{ANALYSIS}

The main vibrating system is considered as a damped-SDOF one of mass $\mathrm{M}_{1}$, damping coefficient $\mathrm{c}_{1}$ and stiffness $\mathrm{k}_{1}$. Its vibration is excited by a harmonic force F0sinwt. An undamped absorber-harvester of mass $\mathrm{M}_{2}$ and stiffness $\mathrm{k}_{2}$ is attached to the main system. A line diagram of the dynamic system is shown in Fig.1

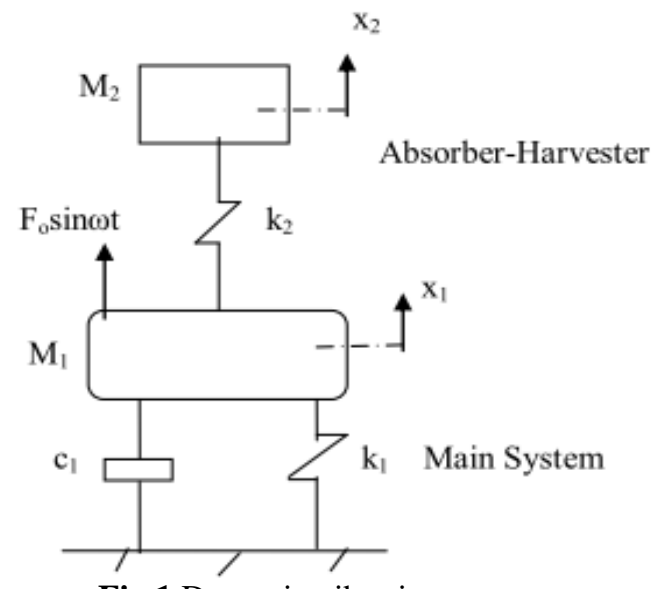

Fig.1 Dynamic vibrating system

With the absorber-harvester attached to the main system it becomes a damped two-degree of freedom one having a differential equation of the form:

$$
\mathbf{M x} \mathbf{x}^{\prime}+\mathbf{C \mathbf { x } ^ { \prime }}+\mathbf{K x}=\mathbf{F}_{\mathrm{o}} \mathrm{e}^{\mathrm{j} \omega \mathrm{t}}
$$

Where

$\mathbf{M}=$ system mass matrix

$\mathbf{C}=$ system damping matrix

$\mathbf{K}=$ system stiffness matrix

And $\mathbf{x}=$ vibration motion vector

The steady-state solution of Eq. 1 is:

$$
\mathbf{x}=\mathbf{X} \mathrm{e}^{\mathrm{j} \omega \mathrm{t}}
$$

Where $\mathbf{X}$ is the phasor of the vibration amplitude vector

Combining Eqs. 1 and 2 gives the peak amplitude ratio of the two masses as:

$$
X_{l} / X_{0}=\frac{1-\omega_{\mathrm{r}}^{2}}{\sqrt{ }\left\{\left[\left(1-\omega_{\mathrm{r}}^{2}\right)\left(1+\mu \beta^{2}-\beta^{2} \omega_{\mathrm{r}}^{2}\right)-\mu \beta^{2}\right]^{2}+\left[2 \zeta \omega_{\mathrm{r}} \beta\left(1-\omega_{\mathrm{r}}^{2}\right)\right]^{2}\right\}}
$$

And

$$
X_{2} / X_{0}=\frac{1}{\sqrt{\left\{\left[\left(1-\omega_{r}^{2}\right)\left(1+\mu \beta^{2}-\beta^{2} \omega_{r}^{2}\right)-\mu \beta^{2}\right]^{2}+\left[2 \zeta \omega_{\mathrm{r}} \beta\left(1-\omega_{\mathrm{r}}^{2}\right)\right]^{2}\right\}}}
$$

Where:

$\mu=$ Mass ratio $=\mathrm{M}_{2} / \mathrm{M}_{1}$

$\beta=\omega_{22} / \omega_{11}$

$\omega_{11}=$ Natural frequency of the main system

$\omega_{22}=$ Natural frequency of the absorber system

$\omega_{\mathrm{r}}=$ Exciting frequency ratio $=\omega / \omega_{22}$

$\zeta=$ Damping ratio of the main system

$\mathrm{X}_{\mathrm{o}}=$ Static deflection corresponding to $\mathrm{F}_{\mathrm{o}}=\mathrm{F}_{\mathrm{o}} / \mathrm{k}_{1}$

\section{OPTIMIZING THE ABSORBER-HARVESTER PARAMETERS}

\subsection{Design Parameters}

The absorber-harvester parameters are:

- $\quad$ Its mass ratio, $\mu$

- $\quad$ Its natural frequency ratio, $\beta$

\subsection{Optimization Technique}

The MATLAB optimization toolbox is used to solve this optimization problem through using its command "fmincon" [16]. It is a technique used to solve multi-dimensional constrained optimization problems.

\subsection{Objective Function}

Here, we are interested to minimize the vibration amplitude of the main system and to increase the vibration amplitude of the absorber-harvester to increase the energy generation. Both are contradicting objectives which can be achieved using an objective function $\mathrm{f}$ in the form:

$$
f=\left(X_{1} / X_{o}\right)^{2}+\left(X_{2} / X_{o}\right)^{-2}
$$

\subsection{Constraints}

First of all, the optimization problem is considered as an unconstrained one. However, a negative mass ratio was obtained. Therefore, it was essential to transfer to the constrained optimization approach by imposing a constraint on the mass ratio in the form.

$$
\mathrm{C}_{1}=\mu_{\min }-\mu
$$

Where $\mu_{\min }$ is a minimum desired level for the absorberharvester mass ratio. It was set to 0.05 .

\section{OPTIMIZATION RESULTS}

\subsection{Absorber-Harvester Natural Frequency Ratio}

The optimal absorber-harvester natural frequency, $\omega_{22} / \omega_{11}$ is function of the optimal mass ratio $\mu$ and main system damping ratio $\zeta$ as shown in Fig. 2 . 


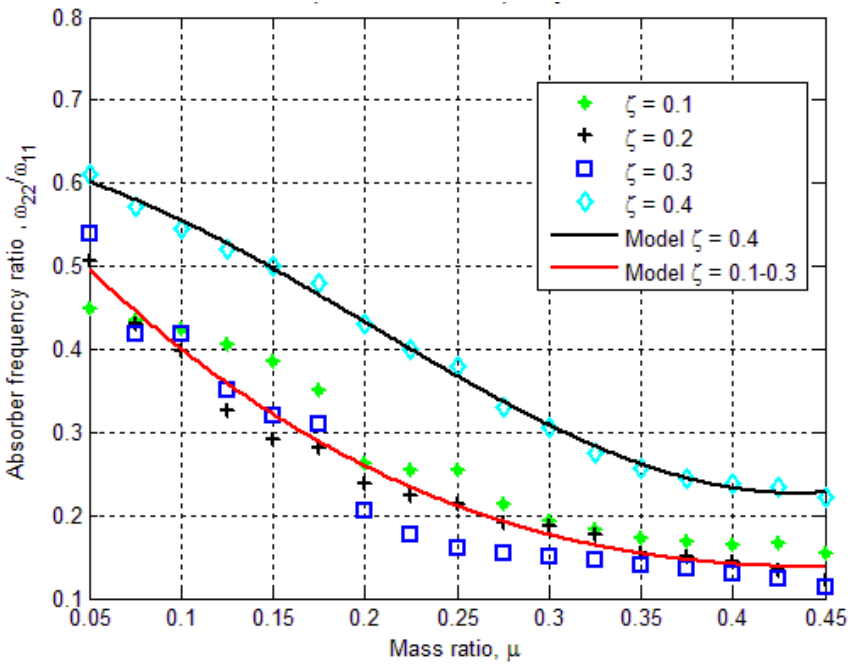

Fig.2 Optimal absorber-harvester natural frequency ratio

The optimal values of $\omega_{22} / \omega_{11}$ for 0.4 damping ratio are greater than those for the other damping ratio values from 0.1 to 0.3 . The optimal $\omega_{22} / \omega_{11}$ is related to the optimal mass ratio $\mu$ through:

$$
\omega_{22} / \omega_{11}=8.15356 \mu^{3}-4.946336 \mu^{2}-0.314653 \mu+0.628018
$$

\section{With 0.9966 correlation coefficient}

The other values of the optimal $\omega 22 / \omega 11$ for $0.1,0.2,0.3$ are relatively closer to each other. So, one $3^{\text {rd }}$ order polynomial model was fitted to those optimal data as:

$$
\omega_{22} / \omega_{11}=-1.91657 \mu^{3}+4.09031 \mu^{2}-2.50654 \mu+0.612128
$$

\section{With 0.9440 correlation coefficient}

\subsection{Harvesting Frequency}

The optimal frequency spectrum of the vibrating system as a $2 \mathrm{DOF}$ one at 0.3 damping ratio and 0.125 mass ratio is shown in Fig.3.

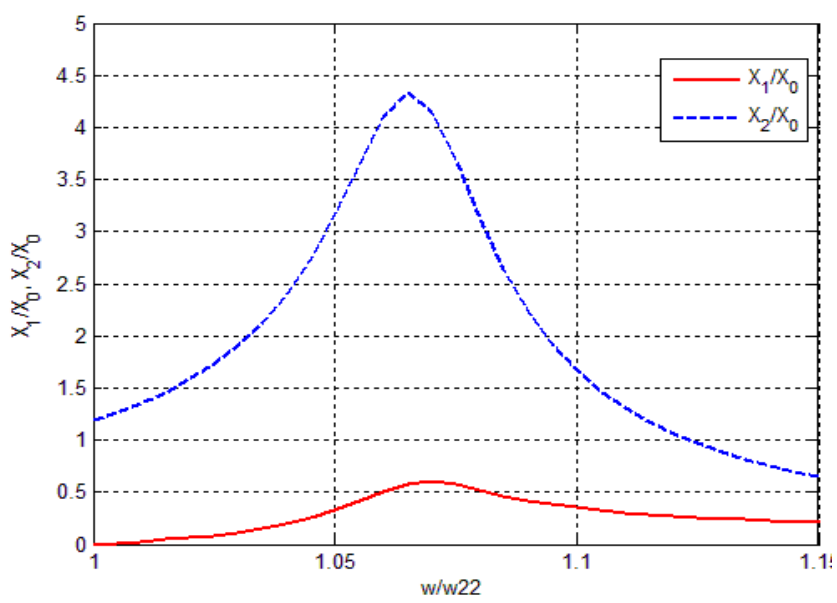

Fig.3 Optimal frequency spectrum for $\zeta=0.3$ and $\mu=0.125$.
The vibration amplitude ratio drops to zero at $\omega / \omega_{22}=0$ (tuning condition of the absorber-harvester). The absorberharvester amplitude ratio (blue curve in Fig.3) increases to a maximum value at a frequency I call it the "harvesting frequency", $\omega_{\mathrm{H}}$ slightly above the tuning condition.

The harvesting frequency ratio $\omega_{\mathrm{H}} / \omega_{22}$ for different main system damping ratio in the range $0.1 \leq \zeta \leq 0.4$ and mass ratio in the range $0.05 \leq \mu \leq 0.45$ is shown in Fig. 4 .

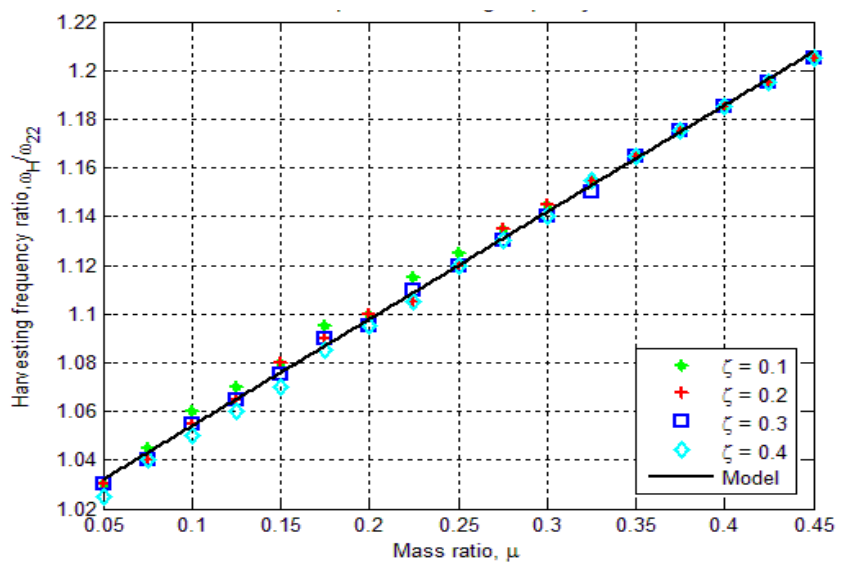

Fig.4 Optimal harvesting frequency ratio

The values are very close to each other regarding the damping ratio effect and very close to a straight line relation. This means that the harvesting frequency is independent of the main system damping ratio and depends only on the absorber-harvester mass ratio. Mathematically:

$$
\omega_{\mathrm{H}} / \omega_{22}=0.438848 \mu+1.010141
$$

With 0.9989 correlation coefficient

\subsection{Main System Vibration Amplitude at}

\section{Harvesting Frequency}

The vibration amplitude of the main vibrating system at the harvesting frequency is increased than the zero level at the tuning condition. This increase depends on both main system damping ratio and mass ratio of the absorberharvester. This is illustrated in Fig.5.

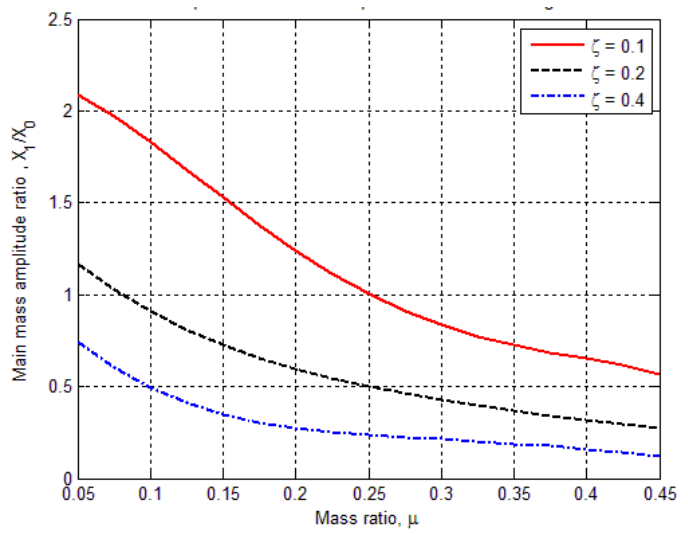

Fig.5 Optimal vibration amplitude ratio of the main system 
The vibration amplitude ratio of the main system decreases with increased damping ratio of the main system and mass ratio of the absorber-harvester.

\subsection{Absorber-Harvester Vibration Amplitude at}

\section{Harvesting Frequency}

This is the second objective of this novel work. This optimal design is proposed to provide relatively high motion of the absorber-harvester mass to be used for energy harvesting using mechanical vibrations. The maximum amplitude ratio of the absorber-harvester mass is shown inFig.6 as function the mass ratio and damping ratio.

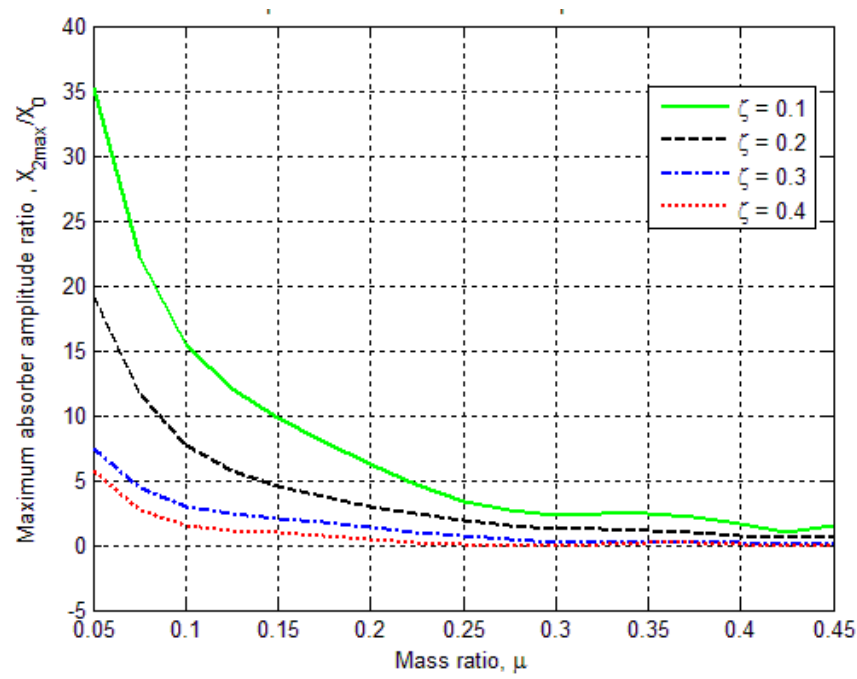

Fig.6 Optimal vibration amplitude ratio of the absorberharvester mass.

The vibration amplitude ratio of the absorber-harvester mass decreases with increased damping ratio of the main system and mass ratio of the absorber-harvester. However, it starts with relatively large values reaching 35 for 0.05 mass ratio and 0.1 damping ratio.

\section{MAIN SYSTEM VIBRATIONS}

The main system alone is a SDOF excited by a constant amplitude harmonic force. When connected to the absorberharvester sub-systems, the whole system becomes 2DOF one. The frequency response of the 2 systems regarding the main system vibrations excited by the same exciting force is shown in Fig. 7 for two sets of parameters.
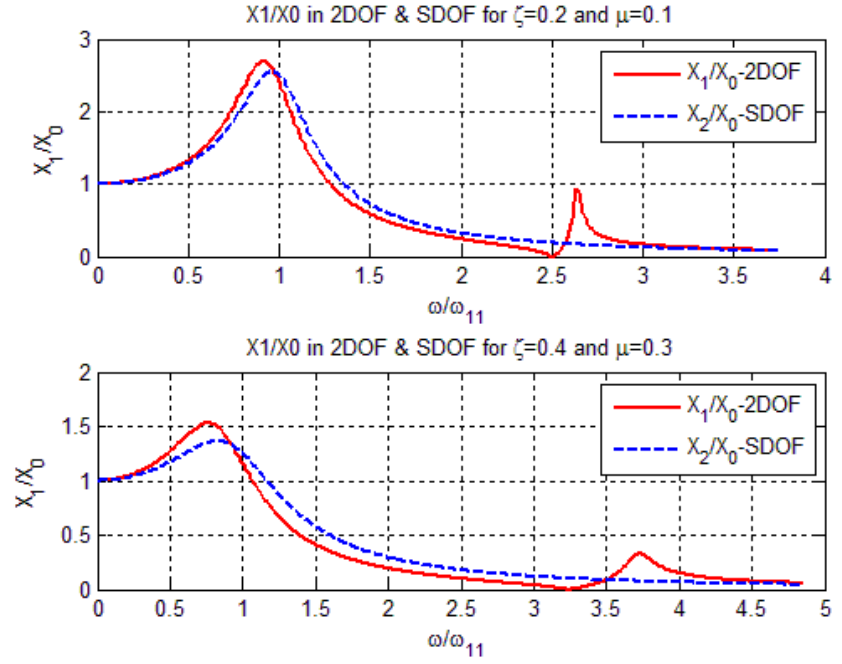

Fig.7 Vibration amplitude ratio of the main vibrating system

The vibration amplitude is plotted against the exciting frequency ratio $\omega / \omega_{11}$ and not against $\omega / \omega_{22}$ as in the analysis presented for the 2DOF system.

It is worth to notice here that the vibration amplitude ratio of the main system with absorber-harvester is less than that without absorber harvester (SDOF) for frequency ration from 1 to 2.5 for the first set of parameters and from 1 to 3.25 for the second set of parameters.

Besides, the vibration amplitude drops to exactly zero at the tuned exciting frequency $\left(\omega=\omega_{22}\right)$.

\section{VIBRATION CONTROL-ENERGY}

\section{HARVESTING}

The procedure for using the scheme proposed in this paper for vibration control and energy harvesting is as follows:

(i) The main vibrating system has a known natural frequency $\omega 11$ and damping ratio $\zeta$..

(ii) For a specific main vibrating system, a proper mass ratio of the absorber-harvester can be selected to satisfy the requirements of the main system amplitude (using Fig.4) and the absorber-harvester vibration amplitude ratio (using Fig.5).

(iii) Using Eq.8 and the assigned mass ratio in step ii, the natural frequency of the absorber-harvester subsystem can be assigned.

(iv) Using steps ii and iii, the parameters of the absorber-harvester ( 2 2 and M2) are ready for calculations.

(v) Now, the harvesting frequency can be obtained using Eq.9.

(vi) If the machine operator is interested only in reducing the machine vibrations, he can tune the machine speed (in variable speed drives) to the natural frequency of the absorber-harvester.

(vii) If the operator is interested in energy harvesting, he can tune the machine speed to the harvesting frequency to attain the maximum possible amplitude of the absorber-harvester mass. 
(viii) These adjustments can be applied automatically in PLC or microprocessor controlled machines.

\section{CONCLUSIONS}

- A novel absorber-harvester was studied in this work to accompany SDOF like machines and vibrating systems.

- A new frequency was defined which is the harvesting frequency.

- It was possible to reduce the vibration amplitude of the main vibrating to zero (eliminating completely its vibrations) by tuning the exciting frequency to the absorberharvester natural frequency.

- At the harvesting frequency, the vibration amplitude of the absorber-harvester mass is maximum depending on the damping ratio of the main system and the absorber mass ratio.

- The vibration amplitude of the absorber-harvester mass increased as the damping ratio of the main system and mass ratio of the absorber decrease.

- The proposed vibration absorber-harvester system is workable for both objectives: vibration reduction and energy generation increase.

\section{ACKNOWLEDGEMENTS}

The author would like to thank his teaching assistants Eng. Ontwan Demetry and Eng. Mohammed AbdelHafiz for their remarks regarding the MATLAB code.

\section{REFERENCES}

[1]. D. Pade and V. Steffen (2000), "Optimization of dynamic vibration absorber over a frequency band", Mechanical Systems and Signal Processing, Vol.14, No.5, pp.679-690.

[2]. S. Miller (2003), "The development of nonlinear elastomeric adaptive tuned vibration absorber", M.Sc. Thesis, The Faculty of Royan University, NJ, USA, October.

[3]. J. Bonsel, R. Fey and H. Nijmeijer (2004), “Application of a dynamic vibration absorber to piecewise linear beam system", Nonlinear Dynamics, Vol.37, pp.227-243.

[4]. P. Varpasuo (2006), "Solution strategies for FPKequation using standard FEM software for diffusion problems", Rakenteiden Mekaniikka, Vol.39, No.1, pp.5-11. [5]. S. Jang et.al. (2007), "A study of the design of a cantilever type multi-DOF dynamic vibration absorber for micro machine tools", $14^{\text {th }}$ International Congress on Sound and Vibration, Cairns, Australia, 9-12 July

[6]. M. Ozkan (2010), "Dynamic response of beams with passive tuned mass dampers", M.Sc. Thesis, Faculty of Purdue University, West Lafayette, Indiana, USA, May.

[7]. G. Liao, X. Gong, C. Kang and S. Xuan (2011), "The design of an active-adaptive tuned vibration absorber based on magneto rheological elastomer and its vibration attenuation performance", Smart Materials and Structures, Vol.20, pp.1-10.

[8]. R. Mirsanei, A. Hajikhani, B. Peykari and J. Hamedi (2012), "Developing a new design for adaptive tuned dynamic vibration absorber based on smart crank-slider mechanism to control undesirable vibrations", International
Journal of Mechanical Engineering and Mechatronics, Vol.1, No.1, pp.80-87.

[9]. Y. Shen and M. Ahmadian (2013), "Nonlinear dynamical analysis on four semi-active dynamic vibration absorbers with time delay", Shock and Vibration, Vol.20, pp.649-663.

[10]. S. Huang and K. Lin (2014), "A new design of vibration absorber for periodic excitation", Shock and Vibration, Vol. 2014, Article ID 571421.

[11]. C. Kim et.al. (2010), "Design and evaluation of a piezoelectric energy harvester produced with a finite element method", Transactions on Electrical and Electronic Materials, Vol.11, No.5, pp.206-211.

[12]. S. Li, J. Yuan and H. Lipson (2011), “Ambient wind energy harvesting using cross-flow fluttering", Journal of Applied Physics, Vol.109, Article Number 026104.

[13]. K. Han, D. Shin and Y. Choi, (2012), "Research of wideband vibration system of energy harvest system for wireless system", International Journal of Emerging Technology and Advanced Engineering, Vol.2, No.12, pp.49-53.

[14]. Y. Wang, C. Chen and C. Sung (2013), "System design of a weighted pendulum-type electromagnetic generator for harvesting energy from a rotating wheel", IEEE/ASME Transactions Mechatronics, Vol.18, No.2, April, pp.754-763.

[15]. S. Nithiya, K. Sadhuna and A. Saravanan (2014), "Energy harvesting using oscillating pendulum", International Journal for Research and Development in Engineering, Special Issue, Methods Enriching Power and Energy Development, pp.17-19.

[16]. P. Venkataraman (2009), “Applied optimization with MATLAB programming”, J. Wiley and Sons.

\section{BIOGRAPHIE}

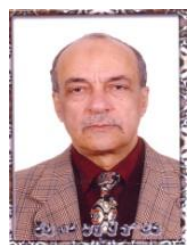

Prof. Galal Ali Hassaan, Emeritus professor of System Dynamic and Automatic Control, Department of Mechanical Design \& Production, Faculty of Engineering, Cairo University. Research in Automatic Control, Mechanical Vibrations and Mechanism 